\title{
Study and Simulation of the Model of Stability Measurement for Integrated Semiconductor Equipment
}

\author{
Xue Fang \\ Department of Physics and Electrical Engineering \\ Handan College \\ Handan 056005, China \\ hdxyxf2006@163.com
}

\author{
Wang Haifei \\ Department of Physics and Electrical Engineering \\ Handan College \\ Handan 056005, China
}

\begin{abstract}
Performance analysis and capacity prediction of integrated semiconductor equipment is a very difficult task, it is a effective way to solve this problem by setting up a model of equipment performance measurement. This paper details the composition and related operation principle of parallel integrated semiconductor equipment. This paper also deriving a set of stability measurement model, we call it Throughput Model, for integrated semiconductor equipment according to its operation principle, which reflect the relationship between stability and output error. The model can effectively calculate the stability of equipment and help system design.
\end{abstract}

Keywords-Parallel; Integrated Semiconductor Equipment Model of Stability; Simulation

\section{INTRODUCTION}

Semiconductor Equipment is a type of important equipment in current semiconductor manufacture. According to SEMI standard E21-96, integrated semiconductor equipment is a kind of semiconductor manufacture equipment which integrates and mechanical combined multiple manufacture module, transmission module and wafer bearing chamber. In semiconductor equipment, there are several wafer processing modes, such as serial, parallel, mixture and re-loadable wafer stream modes. It's difficult to analyze the performance and predict the yield of the integrated semiconductor equipment due to the characteristics of the equipment and the complex of the processing. The integrated semiconductor equipment model can effectively analyze the relationship among yield, equipment configuration and wafer stream modes to predict the equipment yield and optimize the system configuration.

Perkinson draw the sequence chart of the parallel integrated semiconductor equipment processing and deduced the theoretic equipment model to predict the maximum throughput based on single-beam serial integrated semiconductor equipment and serial-parallel mixture wafer stream modes[1-2]. Morrison and Martin calculated the equipment average cycle time using queuing theory which aimed at the serial wafer processing modes of integrated semiconductor equipment[3]. Srinivasan applied Petri network to establish semiconductor equipment processing model and analyzed the model by state cycle analysis model to calculate the stable yield[4]. Wu N Q considered constrained integrated semiconductor equipment model and did much work on dispatch analysis using Petri network method[5]. The researcher analyzed the system dispatch and deduced a analytical dispatch algorithm to determine the periodical dispatch scheme based on the model and time constrains.

Petri network model performance analysis is difficult and can cause state space explosion problem. However, Perkinson or Morrison didn't carry research on the throughput[6] of parallel integrated equipment. This paper establishes the throughput model for the parallel integrated semiconductor equipment and analyzes the performance.

\section{INTRODUCTION OF THE INTEGRATED SEMICONDUCTOR EQUIPMENT}

The integrated semiconductor equipment usually consist of several processing chambers, one or two wafer bearing chambers and a transmission module based on robotic hand. The processing module is responsible for processing wafers. There are one or two bearing plates in robotic hand which are responsible for loading and unloading and moving the wafers. The wafer bearing chamber is responsible for storing the pending and processed wafers. For a N-chamber parallel integrated semiconductor equipment, the function of each chamber is the same. There are five states-initial temporary, stable, finish temporary and unload states.

(1) Loading Stage. A batch of wafers firstly is put in the wafer bearing chamber, and then the chamber is pumped into vacuum space. This stage is the system loading stage and the required time is system loading time.

(2) Initial temporary stage. After system loading the wafer bearing chamber is in vacuum. The robotic hand can unload the wafers in the chamber and transfer them to the processing chambers. The system enters initial temporary stage.

(3) Stable stage. The system enters the stable stage when there are wafers in all of the processing chambers. The system in this stage has the periodical characteristic and stays the longest time. Thus the characteristics in stable stage can reflect the system characteristics. In the stable stage, the time which is the time interval of two successively finished wafers loaded in the wafer bearing chambers is called wafer basic period, represented by WFP.

(4) Finish temporary stage. The processing lost the periodical characteristics when the amounts of pending 
wafers are less the chamber number $\mathrm{N}$. The system enters the finish temporary stage.

(5) Unload stage. When all of the wafers are processed completely and loaded to wafer bearing the unload stage begins. At this time the wafer bearing chambers are pumped into air to atmosphere pressure. The robotic hand takes away all of the wafers from the wafer bearing chambers and the stage end.

Normally the time which the system in stable stage is longest and has the periodical characteristic. Thus the characteristics in stable stage can reflect the system characteristics. In the stable stage, the time which is the time interval of two successively finished wafers loaded in the wafer bearing chambers is called wafer basic period, represented by WFP, which is one the most important index to evaluate the equipment.

\section{STABLE MEASUREMENT MODEL DESIGN}

In order to evaluate the stability of the performance, a stable measurement model is designed. The method is as follows.

Consider the system

$$
\begin{aligned}
& E x(t)=A_{1} x(t)+A_{2} x(t-h)+B \omega(t) \\
& y(t)=C x(t) \\
& \omega(t)=-\gamma(H x(t))
\end{aligned}
$$

In the equations $x \in R^{n}, y \in R^{m}, \omega \in R^{m}$, constant matrix $E 、 A_{1} 、 A_{2} \in R^{n \times n}, B \in R^{n \times m}$, $C 、 H \in R^{m \times n}$ and multi-variable nonlinear function $\gamma(\cdot): R^{m} \rightarrow R^{m}$ and $\gamma(u)=\left(\begin{array}{c}\gamma_{1}\left(u_{1}\right) \\ \vdots \\ \gamma_{m}\left(u_{m}\right)\end{array}\right)$, system observer is in the following format:

$$
\begin{aligned}
& \hat{E x}(t)=A_{1} \hat{x}(t)+A_{2} \hat{x}(t-h)+K(\hat{y}(t)- \\
& y(t)_{{ }_{+}} B \hat{\omega}(t) \\
& \hat{y}(t)_{=} C \hat{x}(t) \\
& \hat{\omega}(t)_{==} \gamma_{\left.\left(S_{(} \hat{y}(t)_{-} y(t)\right)+H \hat{x}(t)\right)}
\end{aligned}
$$

The error observer system is:

$$
\begin{aligned}
& E e(t)_{=}\left(A_{1}+K C\right) e(t)+A_{2} e(t-h)_{+} \\
& B\left(\omega(t)_{-} \hat{\omega}(t)_{)},\right. \\
& \left.\hat{\omega}(t)_{=-} \gamma_{(-} S C e(t)_{)+} H \hat{x}(t)\right),
\end{aligned}
$$

In the equations, $e(t)=x(t)-\hat{x}(t)$ is represented the actual wide meaning error and estimated wide system sates errors.

Out goal is to find corresponding dimension matrix $\mathrm{S}$ and $\mathrm{K}$ when $t \rightarrow \infty$ the state error $e(t) \rightarrow 0$. At this time we assume the system solutions in finite time is finite value. Suppose $u(t)=H x(t), v(t)=S(\hat{y}(t)-y(t))+H \hat{x}(t))$, the observation errors can be regarded as nonlinear feedback and regards $\gamma(u(t))-\gamma(v(t))$ as the function of $t$ and

$$
z(t)=u(t)-v(t)=(H+S C) e(t) .
$$

Assuming $\psi(t, z(t))=\gamma(u(t))-\gamma(v(t))$, it can be rewritten as,

$$
\begin{aligned}
& E e(t)=\left(A_{1}+K C\right) e(t)+A_{2} e(t-h)+B \Omega(t) \\
& z(t)_{=}(H+S C) e(t), \\
& \Omega(\mathrm{t})==\psi(t, z(t)) . \\
& \psi(t, z(t))_{\text {satisfies the properties of multi-variable, thus }} \\
\text { for } & \forall 1 \leq i \leq m \\
& \psi_{i}(t, z(t))_{=} \gamma_{i}(u(t))_{-} \gamma_{i}(v(t)) \\
= & \gamma_{i}\left(u_{i}(t)\right)_{-} \gamma_{i}\left(v_{i}(t)\right)_{=} \psi_{i}\left(t, z_{i}(t)\right) \\
= & \int_{0}^{1}\left[\frac{\partial \gamma}{\partial s}\right]_{s=u_{i}+\xi\left(v_{i}(t)-u_{i}(t)\right)}\left(u_{i}(t)-v_{i}(t)\right) d \xi \text {, thus the }
\end{aligned}
$$
components $\psi_{i}(.,$.$) must satisfy following sector constrains$ $\psi_{i}\left(t, z_{i}(t)\right)\left[\psi_{i}\left(t, z_{i}(t)\right)-b_{i} z_{i}(t)\right] \leq 0$, the matrix is written as $K=\operatorname{diag}\left\{b_{1}, \ldots, b_{m}\right\}>0$ with $\psi^{T}(t, z(t))_{[} \psi(t, z(t))_{-} K z(t)_{]} \leq 0$, the convergence of the observer is the conclusion of theorem 1 .

At this time, state vector $x(t)$ is replaced by $e(t)$, output vector $y(t)$ is replaced by $z(t)$, the coefficient matrix $A_{1}$ is replaced by $A_{1}+K C$ and $C$ is replaced by $H+S C$. According to theorem 3 , there exists positive definite matrix $P 、 Q 、 R$ make $\operatorname{LMI}(9)\left(\Lambda_{<0)}\right.$. S can be solved by LMI(7) to get stability results.

\section{EXPERIMENT ANALYSIS}

In order to verify the validity of the method the comparison experiment is applied for the stability experiment of some specific semiconductor system. The simulation is finished by a semiconductor equivalent circuit. The experimental circuit is as figure 1 . 


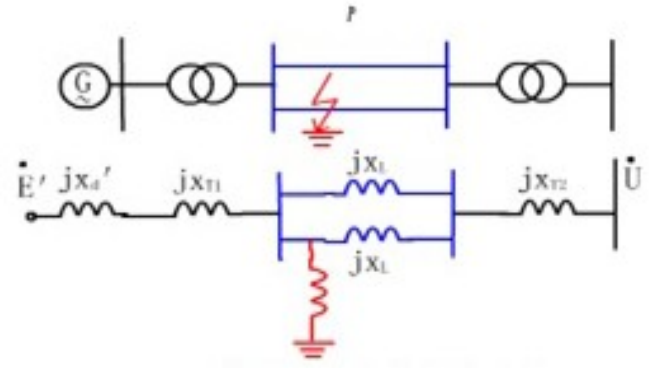

Figure 1. System analog circuit scheme

The following is the comparison of the stable effect diagrams of the semiconductor system measured by two different methods. The comparison diagrams are as figure 2 .

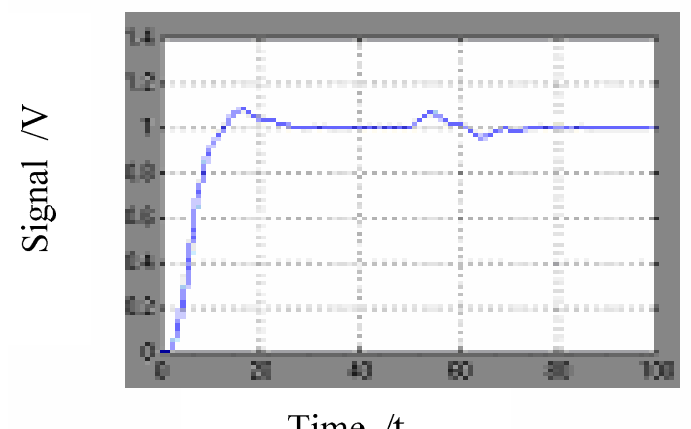

Time $/ t$

Figure 2. The stable trend diagram of the algorithm proposed in this paper

In figure 2 the system temporary stable effect is obvious. The algorithm reduces the measurement model instability caused by the reference to ensure the accuracy of the results which has some practical value.

\section{CONCLUSIONS}

This paper detailed introduces the components and mechanism of parallel integrated semiconductor equipment. It deduces the integrated semiconductor stability measurement model to reflect the relationship between the stability and the output error. The throughput model can calculate the equipment stability effectively and aid the system design which can evaluate the system stability quickly.

\section{ACKNOWLEDGMENT}

Chinese book Classification Code: TP202

Document code: A

The paper is supported by the Research and Development Program for Science and Technology of Handan (Grant No. : 1021140118-7)

\section{REFERENCES}

[1] Perkinson T L, McLarty P K, Gyurcsik R S, Cavin III R K. Singlewafer cluster tool performance: an analysis of throughput[J]. IEEE Transactions on Semiconductor Manufacturing, 1994, 7(3): 369-373.

[2] Perkinson T L, Gyurcsik R S, McLarty P K. Single-wafer cluster tool performance: an analysis of the effects of redundant chambers and revisitation sequences on throughput[J]. IEEE Transactions on Semiconductor Manufacturing, 1996, 9(3): 384-400.

[3] MORRISON J R, MARTIN D P. Performance evaluation of photolithography cluster tools[J]. OR Spectrum, 2007, 29(3): 375-389.

[4] SRINIVASAN R S. Modeling and performance analysis of cluster tools using Petri nets[J]. IEEE Trans. Semiconduct. Manufact.1998,11(3): 394-403.

[5] Wu N Q, Zhou M C, Analysis of wafer sojourn time in dual-arm cluster tools with residency time constraint and activity time variation[J]. IEEE Transactions on Semiconductor Manufacturing, 2010, 23(1): 53-64.

[6] Cho J H, Ryu S J, Chae S K. Throughput analysis for cluster tool under transfer bound condition and its application to dry etch equipment[C]. 2003 IEEE International Symposium on Semiconductor Manufacturing, 2003, 289-292 